\title{
Fixed bandwidth asymptotics for the studentized mean of fractionally integrated processes*
}

\author{
Javier Hualde \\ Universidad Pública de Navarra
}

\author{
Fabrizio Iacone ${ }^{\dagger}$ \\ University of York
}

October 7, 2016

\begin{abstract}
We consider inference for the mean of a general stationary process based on standardizing the sample mean by a frequency domain estimator of the long run variance. Here, the main novelty is that we consider alternative asymptotics in which the bandwidth is kept fixed. This does not yield a consistent estimator of the long run variance, but, for the weakly dependent case, the studentized sample mean has a Student- $t$ limit distribution, which, for any given bandwidth, appears to be more precise than the traditional Gaussian limit. When data are fractionally integrated, the fixed bandwidth limit distribution of the studentized mean is not standard, and we derive critical values for various bandwidths. By a Monte Carlo experiment of finite sample performance we find that this asymptotic result provides a better approximation than other proposals like the test statistic based on the Memory Autocorrelation Consistent (MAC) estimator of the variance of the sample mean.
\end{abstract}

Keywords: long run variance estimation, fractional integration, large- $m$ and fixed- $m$ asymptotic theory.

JEL classification: C32

*We thank Jia Chen and Laura Coroneo for helpful suggestions and discussions. Javier Hualde's research is supported by the Spanish Ministerio de Economía y Competitividad through project ECO201564330-P.

${ }^{\dagger}$ Corresponding author: Fabrizio Iacone, Department of Economics and Related Studies, University of York, Heslington, York, YO10 5DD, UK. 


\section{INTRODUCTION}

We consider inference for the mean of a covariance stationary time series with autocorrelation of unspecified nature. This problem has been widely studied for weakly dependent processes (whose spectral density is finite and nonzero), where robust inference can be obtained by standardizing the sample average by the long run variance: this is usually unknown, but it can be estimated by a range of techniques based on either weighted autocovariances or weighted periodograms, see, e.g., Priestley (1981).

In the present paper we emphasize the use of frequency domain techniques. In this setting, the simplest estimator of the long run variance can be obtained by direct averaging periodograms evaluated at the first $m$ Fourier frequencies (which corresponds to using the Daniell kernel), where $m$ is known as bandwidth. When discussing the limiting properties of this estimator, it is routinely assumed that $m \rightarrow \infty$, although at a rate slower than the sample size $T$, so that the band $m / T$ is degenerating to 0 . Throughout, we will denote the assumption $m \rightarrow \infty$ as the large- $m$ approach. Here, we will consider instead an alternative strategy to derive the asymptotic properties in which $m$ is kept fixed. The motivation is that in any practical situation a finite $m$ is used, so letting the asymptotic distribution depend on a fixed $m$ might yield a better approximation to the sampling distribution of the corresponding test statistic. This approach will be denoted as fixed- $m$, and can be seen as a frequency domain analogue to the fixed- $b$ approach for time domain estimators of the long run variance. The fixed- $b$ strategy has been also applied to provide a more accurate limit approximation to the sampling distribution of the studentized mean (see, e.g., Kiefer and Vogelsang, 2002, 2005, Jansson, 2004, Sun, Phillips and Jin, 2008, McElroy and Politis, 2012, 2013).

Despite the analogies, the fixed- $m$ and fixed- $b$ limits are different and, as we show in the paper, in the leading case of weakly dependent processes, the fixed- $m$ approach does not require the simulation of a null limit distribution (which is found to be a Student- $t$ with $2 m$ degrees of freedom, $t_{2 m}$ ). However, weak dependence is just a particular case of the general type of dependence we allow for in the paper, which is captured by the so-called fractional processes. This includes the long memory and antipersistent situations, with positive and negative memories, respectively, where the fixed- $m$ limit of the standardized mean is not standard and we derive critical values for various bandwidths.

The following section presents the studentized mean and discusses its large- $m$ and fixed- $m$ limits. In Section 3, we compare the large- $m$ and fixed- $m$ limiting approximations to the sampling distribution of the studentized mean by a Monte Carlo experiment. Finally, in Section 4 we conclude. Proofs are given in the Appendix.

\section{LARGE- $m$ AND FIXED- $m$ LIMITS OF THE STUDENTIZED MEAN}


We consider the time series $x_{1}, \ldots, x_{T}$, observed from the stationary process $x_{t}:=\mu+$ $u_{t}$, where $E\left(u_{t}\right)=0$ and $u_{t}$ may be subject to a general type of dependence characterized in Assumptions 1 and 2 below.

Assumption 1. Let $\eta_{t}=A(L) \varepsilon_{t}:=\sum_{j=0}^{\infty} A_{j} \varepsilon_{t-j}$ where $L$ is the usual lag operator. The weights $\left\{A_{j}\right\}$ are such that $A(1)^{2}>0$ and $\sum_{l=0}^{\infty} l\left|A_{l}\right|<\infty$ and $\varepsilon_{t}$ is an independent, identically distributed (i.i.d.) sequence with $E\left(\varepsilon_{t}\right)=0, E\left(\varepsilon_{t}^{2}\right)=1$.

Assumption 2. Let $\Delta_{t}^{(\delta)}:=\Gamma(t+\delta) /(\Gamma(\delta) \Gamma(t+1)), \Gamma(\cdot)$ denoting the Gamma function, such that $\Gamma(0):=\infty$ and $\Gamma(0) / \Gamma(0):=1$, and $u_{t}:=\sum_{s=-\infty}^{t} \Delta_{t-s}^{(\delta)} \eta_{s}, \quad \delta \in$ $(-1 / 2,1 / 2)$.

Assumption 1, 2 imply that, in general, $u_{t}$ is a Type 1 fractionally integrated process. We consider inference on $\mu$ when the dependence structure of $u_{t}$ is not known. In this case, the sample mean $\bar{x}:=T^{-1} \sum_{t=1}^{T} x_{t}$, is a natural estimator of $\mu$ and, if $u_{t}$ is weakly dependent, that is $u_{t}=\eta_{t}$ (or $\left.\delta=0\right)$ and regularity conditions are met, inference on $\bar{x}$ can be based on the Central Limit Theorem (CLT)

$$
\sqrt{T}(\bar{x}-\mu) / \sigma \rightarrow_{d} N(0,1)
$$

where $\sigma^{2}:=A(1)^{2}$ is typically known as long run variance. In practice $\sigma^{2}$ is unknown, but a large number of semiparametric techniques are available to estimate it consistently, see, e.g., Priestley (1981). Letting $w_{x}(\lambda):=(2 \pi T)^{-1 / 2} \sum_{t=1}^{T} x_{t} e^{i \lambda t}$ be the Fourier transform of $x_{t}$ and the periodogram $I(\lambda):=\left|w_{x}(\lambda)\right|^{2}$, the Daniell kernel provides a very simple estimator of $\sigma^{2}, \widehat{\sigma}^{2}:=2 \pi \frac{1}{m} \sum_{j=1}^{m} I\left(\lambda_{j}\right)$, where $\lambda_{j}:=2 \pi j / T$. Feasible inference is then conducted using the statistic

$$
\tau:=\sqrt{T}(\bar{x}-\mu) / \widehat{\sigma}
$$

When $m \rightarrow \infty, m / T \rightarrow 0$ and given other regularity conditions, $\widehat{\sigma}^{2}$ is consistent, and it can be substituted in (1) without altering the limit. To derive the fixed- $m$ asymptotic distribution of $\widehat{\sigma}^{2}$, where $m$ is kept fixed, we need to strengthen the moment conditions on $\varepsilon_{t}$.

Assumption 3. There is $q$ such that $E\left(\left|\varepsilon_{t}\right|^{q}\right)<\infty$ with $q \geq \max (2,2 /(1+2 \delta))$.

Remark 1. Under Assumptions 1-3, the following Functional Central Limit Theorem (FCLT) for fractional process holds: for $r \in[0,1]$, as $T \rightarrow \infty$,

$$
\frac{1}{T^{1 / 2+\delta}} \sum_{t=1}^{\lfloor r T\rfloor} u_{t} \Rightarrow \Sigma_{\delta} W_{\delta+1}(r)
$$


where $W_{\delta+1}(r)$ is a Type I fractional Brownian motion, as defined in Mandelbrot and Van Ness (1968), $\Sigma_{\delta}^{2}:=\sigma^{2} \Gamma(1-2 \delta) /[(1+2 \delta) \Gamma(1+\delta) \Gamma(1-\delta)]$ and $\lfloor\cdot\rfloor$ denotes integer part. For further details see Theorems 2.1 and 2.2 of Wang, Lin and Gulati (2003). When $\delta=0$ the limit (3) encompasses the standard convergence to the standard Brownian motion. Let $\widehat{W}_{\delta+1}(r):=W_{\delta+1}(r)-r W_{\delta+1}(1)$ and

$$
Q_{\delta}(j):=\left\{\left(2 \pi j \int_{0}^{1} \sin (2 \pi j r) \widehat{W}_{\delta+1}(r) d r\right)^{2}+\left(2 \pi j \int_{0}^{1} \cos (2 \pi j r) \widehat{W}_{\delta+1}(r) d r\right)^{2}\right\}
$$

Our key result is Lemma 1 below.

Lemma 1. Under Assumption 1-3, for fixed $j=1, \ldots, m$, as $T \rightarrow \infty$,

$$
T^{-2 \delta} 2 \pi I\left(\lambda_{j}\right) \rightarrow_{d} \Sigma_{\delta}^{2} Q_{\delta}(j)
$$

By Lemma 1 and (3) we can establish the following theorem.

Theorem 2. Under Assumption 1-3, for fixed $m$, as $T \rightarrow \infty$,

$$
\tau \rightarrow_{d} \frac{W_{\delta+1}(1)}{\sqrt{\frac{1}{m} \sum_{j=1}^{m} Q_{\delta}(j)}}
$$

When $\delta=0$, it is well known that, under regularity conditions, the joint distribution of $2 \pi I\left(\lambda_{j}\right), j=1, \ldots, m$, converges to that of $m$ independent $2^{-1} \sigma^{2} \chi_{2}^{2}$ variates (see, e.g., Theorem 13 and pp. 225, 226 of Hannan, 1970). Then, by the continuous mapping theorem and exploiting also the asymptotic independence of $I(0)$ and $I\left(\lambda_{j}\right), j=1, \ldots, m$, it is straightforward to derive

Corollary 3. Under Assumption 1, 2 and $\delta=0$, for fixed $m$, as $T \rightarrow \infty$,

$$
\tau \rightarrow{ }_{d} t_{2 m}
$$

Remark 2. In related settings, the Student- $t$ limiting distribution has already been posed by Sun (2013) (Theorem 3.1) and Müller (2014). In particular, our Corollary 3 justifies formally the heuristic discussion of Müller (2014, p.314), who anticipated that, under weak dependence, taking into account the uncertainty in $\widehat{\sigma}^{2}$ instead of relying on consistency arguments, leads to a Student- $t$ limiting result instead of the traditional $N(0,1)$ limit. Thus our Theorem 2, which generalizes this result allowing also for long memory and antipersistency, encompasses Müller's (2014) claim.

Remark 3. Asymptotic expected values and correlation of periodograms are derived in Hurvich and Beltrao (1993), where a limit distribution of the periodogram for the 
Gaussian case is also given. Asymptotics for the Fourier transforms of possibly fractionally integrated processes are also given in Chen and Hurvich (2003) and Lahiri (2003); see also Deo (1997). We find that (4), where the limiting distribution depends mainly on integrals involving the fractional Brownian bridge $\widehat{W}_{\delta+1}(r)$, provides an easy way to simulate quantiles for the fixed- $m$ limit distribution of $\tau$ when $\delta \neq 0$. This in turn allows for a simple practical application of the limit distribution in (5) for testing, which we view as one of the main contributions of our work.

Remark 4 Theorem 1 provides results for Type I fractionally processes. Results for Type II fractionally integrated processes may be obtained in the same way, just replacing the type of fractional Brownian motion in the limit.

Remark 5. Theorem 2 and Corollary 3 relate directly to results in McElroy and Politis (2012, 2013), who proposed to studentize the sample mean of long, short and negative memory processes by means of a weighted covariance estimator of the long run variance. The similarity between this estimator and the smoothed periodogram $\widehat{\sigma}^{2}$ could be discussed more in detail. Defining the sample covariance of $x_{t}$,

$c(l):=T^{-1} \sum_{t=1}^{T-l}\left(x_{t}-\bar{x}\right)\left(x_{t+l}-\bar{x}\right)$, for $l \geq 0 ;:=T^{-1} \sum_{t=1-l}^{T}\left(x_{t}-\bar{x}\right)\left(x_{t+l}-\bar{x}\right)$, for $l<0$,

the weighted covariance estimator of $\sigma^{2}$ is $\widehat{\sigma}_{W C}^{2}:=\sum_{l=-T+1}^{T-1} k(l / M) c(l)$, where, for the Daniell kernel, $k(x):=\sin (\pi x) /(\pi x)$ and $M$ is a bandwidth parameter such that $1 \leq$ $M \leq T$. Then, for $K_{M}(\lambda):=(2 \pi)^{-1} \sum_{|l|<T} k(l / M) e^{-i l \lambda}, \widehat{\sigma}_{W C}^{2}=\int_{-\pi}^{\pi} K_{M}(\lambda) I^{*}(\lambda) d \lambda$, where $I^{*}(\lambda)$ is the periodogram of $x_{t}-\bar{x}$. In the case of the Daniell kernel, the spectral window $K_{M}(\lambda)$ takes value $M /(2 \pi)$ when $-\pi / M \leq \lambda \leq \pi / M$ and 0 otherwise. Thus for $m=\lfloor T /(2 M)\rfloor, \widehat{\sigma}^{2}$ is an approximation of $\widehat{\sigma}_{W C}^{2}$ when $k(x):=\sin (\pi x) /(\pi x)$ is used. Regularity conditions for consistent estimation of $\sigma^{2}$ for weakly autocorrelated series include $M \rightarrow \infty$ but $M / T \rightarrow 0$ as $T \rightarrow \infty$ for $\widehat{\sigma}_{W C}^{2}$, or $m \rightarrow \infty$ but $m / T \rightarrow 0$ as $T \rightarrow \infty$ for $\widehat{\sigma}^{2}$. On the other hand, Kiefer and Vogelsang (2005) consider the limiting distribution of $\widehat{\sigma}_{W C}^{2}$ under the alternative assumption $M=\lfloor b T\rfloor$ for a fixed nonzero $b$ parameter (denoted fixed- $b$ versus the standard small- $b$ approach, where it is assumed $b \rightarrow 0)$. Noting that $M=\lfloor b T\rfloor$ in $\widehat{\sigma}_{W C}^{2}$ corresponds in the approximation $\widehat{\sigma}^{2}$ to taking $m$ as $\lfloor 1 /(2 b)\rfloor$, our fixed- $m$ assumption is then closely related to the fixed- $b$ condition of Kiefer and Vogelsang (2005), which was later employed by McElroy and Politis (2012, 2013) in their discussion of the limiting properties of their studentized mean of general processes which, in particular, encompass our fractionally integrated $x_{t}$. In this sense, Theorem 2 could be viewed as the fixed- $m$ counterpart to the corresponding fixed- $b$ results of McElroy and Politis (2012, 2013), noting that, despite the similarities, the 
fixed- $b$ and fixed- $m$ limits are different.

Remark 6. As it is evident from Theorem 2, in general, the quantiles corresponding to the limiting distribution of $\tau$ must be simulated. We undertook this by approximating integrals in (5) with summations over 10,000 steps. We simulated the distribution for $\delta \in\{-0.49,-0.4,-0.3, \ldots, 0.3,0.4,0.49\}$ and $m \in\{1,2, \ldots, 16\}$ : for each case, we repeated the simulation 10,000 times. In Table 1 we report values $c v$ such that $P(|\tau|>c v)=0.05$. Notice that we also simulated the distribution for $\delta=0$, which is unnecessary, but we made it for completeness to make possible the comparison between the simulated values and the quantiles from the $t_{2 m}$ distribution.

\section{FINITE SAMPLE PERFORMANCE}

We analyse the reliability of the fixed- $m$ approximation in two Monte Carlo exercises. First, we consider a weakly autocorrelated series and study if the fixed- $m$ limit allows for a more precise inference than the standard large- $m$ approximation. We let $u_{t}=$ $\eta_{t}=\phi \eta_{t-1}+\varepsilon_{t}$, for $\varepsilon_{t}$ i.i.d. $N(0,1), \phi=0.5$, and $T=64,256$. We repeated the experiment 10,000 times. For each case, we compute $\tau$ and count the frequency with which the realization $|\tau|$ is above the 95th percentile, using both the $t_{2 m}$ and the $N(0,1)$ distributions. This is like testing the null hypothesis on $\mu$ with theoretical size $5 \%$, so we refer to these values as rejection frequencies. The results are given in Table 2. In all cases, using the $t_{2 m}$ the empirical size is closer to $5 \%$. Note that this is the case even with relatively large values for $m$, for example $m=\sqrt{T}$. Additionally, the size is more precisely approximated the smaller is $m$.

Next, we simulated a Gaussian $\operatorname{ARFIMA}(1, \delta, 0), u_{t}=\Delta^{-\delta} \eta_{t}$ with $\eta_{t}=\phi \eta_{t-1}+\varepsilon_{t}$, $\varepsilon_{t}$ i.i.d. $N(0,1)$, with $\phi=-0.5,0,0.5$, and $\delta=-0.3,0,0.3$, and we computed $\tau$ with $m=1, \ldots, m=16$. Here we compare $\tau$ to Robinson's (2005) test statistic based on a Memory Autocorrelation Consistent (MAC) estimator of the variance of the sample mean (we refer to this statistic as $\theta$ ). Given that assuming knowledge of $\delta$ is unrealistic, we estimated it by the local Whittle estimator (see Robinson, 1995) with bandwidth $J$. Simulation results, such as in Abadir, Distaso and Giraitis (2009), recommend $J=$ $\left\lfloor T^{0.65}\right\rfloor$, so this is the one we adopt. For simplicity, this is also the bandwidth employed in the computation of $\theta$. We considered samples of $T=64,256,1024$, and for each combination we simulate 10,000 repetitions. Finally, $\tau$ is compared to critical values calculated from the estimated $\delta$, which corresponds to the plug-in approach for obtaining critical values (as in, e.g., McElroy and Politis, 2012, 2013).

In Tables 3-5 we present some of the results (those for other combinations of $\delta, \phi$, are available from the authors upon request). We present first the case $\delta=0, \phi=0$, to better appreciate the effect of estimating $\delta$, which causes a relevant size distortion, 
especially in small sample sizes with large bandwidths: the advantage of using fixed- $m$ asymptotic is more important if fractional integration is also considered.

Next we analyse the effect of altering $\delta$ or $\phi$. In summary, changing $\delta$ does not alter much the empirical size, but changing $\phi$ has a more relevant effect, with $\phi>0$ reducing the empirical size (for given $T$ and $\delta$ ), and $\phi<0$ increasing it. We conjecture that these results may be due to the lower order bias in the estimation of $\delta$ caused by the weak autocorrelation in $\eta_{t}$. When $\phi>0, \widehat{\delta}$ is affected by a lower order bias with positive sign: as the quantiles in Table 1 are increasing in $\delta$, this positive bias results in incorrectly selecting for the test based on $\tau$ a critical value that is higher than it should be, thus distorting the size of the test towards 0 . The size distortion for the MAC normalized sample mean is still more relevant. Of course these arguments are reversed in presence of negative autocorrelation, and this is actually the most important reason for concern, as it may generate a relevant size increase, as we indeed find in our experiment. It is however encouraging to see that the size is still reasonable if fixed- $m$ asymptotics instead of large- $m$ (and MAC standardization) are used, especially when $m$ is kept small.

\section{CONCLUSION}

We analysed inference for the mean of a time series with autocorrelation of unspecified nature and that may be fractionally integrated. We considered a standardization of the sample mean obtained averaging the first $m$ periodograms, as it usually done for the estimation of the long run variance using the Daniell kernel, and we derive a different type of asymptotics in which $m$ is kept fixed. We refer to this as fixed- $m$ asymptotics. When the time series is weakly autocorrelated, the studentized sample mean has a fixed- $m$ limit $t_{2 m}$; when $\delta \neq 0$ the limit depends on $\delta$ and must be tabulated. We provide quantiles for it. We compare fixed- $m$ and large- $m$ asymptotics in a Monte Carlo experiment, finding that the former allows for a better approximation of the size in finite samples. We also find that, if fractional integration is allowed for, then large- $m$ asymptotics are more heavily affected by the size distortion in the estimation of $\delta$, thus making the application of fixed- $m$ asymptotics even more preferable.

\section{Appendix.}

Proof of Lemma 1. Let $\widehat{x}_{t}=x_{t}-\bar{x}$. From (3),

$$
\begin{aligned}
& \frac{1}{T^{1 / 2+\delta}} \sum_{t=1}^{\lfloor r T\rfloor} \widehat{x}_{t}=\frac{1}{T^{1 / 2+\delta}} \sum_{t=1}^{\lfloor r T\rfloor}\left(u_{t}-\bar{u}\right)=\frac{1}{T^{1 / 2+\delta}} \sum_{t=1}^{\lfloor r T\rfloor} u_{t}-\frac{1}{T^{1 / 2+\delta}} \sum_{t=1}^{\lfloor r T\rfloor} \bar{u} \\
= & \frac{1}{T^{1 / 2+\delta}} \sum_{t=1}^{\lfloor r T\rfloor} u_{t}-\frac{\lfloor r T\rfloor}{T^{1 / 2+\delta}} \frac{1}{T} \sum_{t=1}^{T} u_{t}=\frac{1}{T^{1 / 2+\delta}} \sum_{t=1}^{\lfloor r T\rfloor} u_{t}-\frac{\lfloor r T\rfloor}{T} \frac{1}{T^{1 / 2+\delta}} \sum_{t=1}^{T} u_{t} \\
\Rightarrow & \Sigma_{\delta}\left(W_{\delta+1}(r)-r W_{\delta+1}(1)\right) .
\end{aligned}
$$


Noting that for $j= \pm 1, \ldots, \pm m, \sum_{t=1}^{T} e^{i \lambda_{j} t}=0$, for $j=1, \ldots, m$, it also holds that $w_{x}\left(\lambda_{j}\right)=(2 \pi T)^{-1 / 2} \sum_{t=1}^{T} \widehat{x}_{t} e^{i \lambda_{j} t}$, and, using $e^{i x}=\cos x+i \sin x$,

$$
\begin{aligned}
I\left(\lambda_{j}\right) & =\frac{1}{2 \pi T}\left(\sum_{t=1}^{T} \widehat{x}_{t}\left(\cos \lambda_{j} t+i \sin \lambda_{j} t\right)\right)\left(\sum_{s=1}^{T} \widehat{x}_{s}\left(\cos \lambda_{j} s-i \sin \lambda_{j} s\right)\right) \\
& =\frac{1}{2 \pi T}\left\{\left(\sum_{t=1}^{T} \widehat{x}_{t} \cos \lambda_{j} t\right)^{2}+\left(\sum_{s=1}^{T} \widehat{x}_{s} \sin \lambda_{j} s\right)^{2}\right\} .
\end{aligned}
$$

By summation by parts,

$$
\begin{aligned}
2 \pi I\left(\lambda_{j}\right)= & \left(\sum_{t=1}^{T-1}\left(\cos \lambda_{j}(t+1)-\cos \lambda_{j} t\right) \frac{1}{\sqrt{T}} \sum_{s=1}^{t} \widehat{x}_{s}\right)^{2} \\
& +\left(\sum_{t=1}^{T-1}\left(\sin \lambda_{j}(t+1)-\sin \lambda_{j} t\right) \frac{1}{\sqrt{T}} \sum_{s=1}^{t} \widehat{x}_{s}\right)^{2},
\end{aligned}
$$

where we used $\sum_{s=1}^{T} \widehat{x}_{s}=0$. Next, by the mean value theorem

$$
\begin{aligned}
& \cos \lambda_{j}(t+1)=\cos \lambda_{j} t-\lambda_{j} \sin \lambda_{j} t+O\left(\lambda_{j}^{2}\right), \\
& \sin \lambda_{j}(t+1)=\sin \lambda_{j} t+\lambda_{j} \cos \lambda_{j} t+O\left(\lambda_{j}^{2}\right),
\end{aligned}
$$

so,

$$
\begin{aligned}
T^{-2 \delta} 2 \pi I\left(\lambda_{j}\right)= & \left(\sum_{t=1}^{T-1}\left(-\frac{2 \pi j}{T} \sin \left(2 \pi j \frac{t}{T}\right)+O\left(\lambda_{j}^{2}\right)\right) \frac{1}{T^{1 / 2+\delta}} \sum_{s=1}^{t} \widehat{x}_{s}\right)^{2} \\
& +\left(\sum_{t=1}^{T-1}\left(\frac{2 \pi j}{T} \cos \left(2 \pi j \frac{t}{T}\right)+O\left(\lambda_{j}^{2}\right)\right) \frac{1}{T^{1 / 2+\delta}} \sum_{s=1}^{t} \widehat{x}_{s}\right)^{2}
\end{aligned}
$$

and, as $T \rightarrow \infty$,

$T^{-2 \delta} 2 \pi I\left(\lambda_{j}\right) \Rightarrow \Sigma_{\delta}^{2}\left\{\left(2 \pi j \int_{0}^{1} \sin (2 \pi j r) \widehat{W}_{\delta+1}(r) d r\right)^{2}+\left(2 \pi j \int_{0}^{1} \cos (2 \pi j r) \widehat{W}_{\delta+1}(r) d r\right)^{2}\right\}$

noting that the contribution due to the $O\left(\lambda_{j}^{2}\right)$ terms is of smaller order.

Proof of Theorem 2. Theorem 2 follows from Lemma 1, (3) and the continuous mapping theorem. 


\section{REFERENCES}

Abadir, K.M., W. Distaso and L. Giraitis, 2009. Two estimators of the long-run variance: beyond short memory. Journal of Econometrics 150, 56-70.

Chen, W.W., and C.M. Hurvich, 2003. Estimating fractional cointegration in the presence of polynomial trends. Journal of Econometrics 117, 95-121.

Deo, R.S., 1997. Asymptotic theory for certain regression models with long memory errors. Journal of Time Series Analysis 18, 385-393.

Hannan, E.J., 1970. Multiple Time Series. Wiley, New York.

Hurvich, C.M., and K.I. Beltrao, 1993. Asymptotics for the low frequency ordinates of the periodogram of a long memory time series. Journal of Time Series Analysis 14, 455-472.

Jansson, M., 2004. The error in rejection probability of simple autocorrelation robust tests. Econometrica 72, 937-946.

Kiefer, N.M., and T.J. Vogelsang, 2002. Heteroskedasticity-autocorrelation robust testing using bandwidth equal to sample size. Econometric Theory 18, 1350-1366.

Kiefer, N.M., and T.J. Vogelsang, 2005. A new asymptotic theory for heteroskedasticity - autocorrelation robust tests. Econometric Theory 21, 1130-64.

Lahiri, S.N., 2003. A necessary and sufficient condition for asymptotic independence of discrete Fourier Transforms under short- and long-range dependence. Annals of Statistics 31, 613-641.

Mandelbrot, B.B., and J.W. Van Ness, 1968. Fractional Brownian motion, fractional noise and applications. SIAM Review 10, 422-437.

McElroy, T., and D.N. Politis, 2012. Fixed- $b$ asymptotics for the studentized mean from time series with short, long or negative memory. Econometric Theory 28, 471-481.

McElroy, T., and D.N. Politis, 2013. Distribution theory for the studentized mean for long, short, and negative memory time series. Journal of Econometrics 177, 60-74.

Müller, U.K., 2014. HAC corrections for strongly autocorrelated time series. Journal of Business and Economic Statistics 32, 311-322.

Priestley, M.B., 1981. Spectral Analysis and Time Series. Academic Press, London.

Robinson, P.M., 1995. Gaussian semiparametric estimation of long range dependence. Annals of Statistics 23, 1630-1661.

Robinson, P.M., 2005. Robust covariance matrix estimation: HAC estimates with long memory/antipersistence correction. Econometric Theory 21, 171180.

Sun, Y., 2013. A heteroskedasticity and autocorrelation robust $F$ test using an orthonormal series variance estimator. Econometrics Reviews 16, 1-26. 
Sun, Y., P.C.B. Phillips and S. Jin, 2008. Optimal bandwidth selection in heteroskedasticityautocorrelation robust testing. Econometrica 76, 175-194.

Wang, Q.,Y.X. Lin, and C.M. Gulati, 2003. Asymptotics for general fractionally integrated processes with applications to unit root tests. Econometric Theory 19, 143-164.

Table 1. Upper $5 \%$ quantile for $|\tau|$ for $m \in\{1, \ldots, 16\}$

\begin{tabular}{|c|cccccccc|}
\hline$\delta \backslash m$ & 1 & 2 & 3 & 4 & 5 & 6 & 7 & 8 \\
\hline-0.49 & 2.725 & 1.646 & 1.332 & 1.185 & 1.076 & 0.996 & 0.941 & 0.895 \\
-0.4 & 2.891 & 1.710 & 1.392 & 1.221 & 1.110 & 1.037 & 0.977 & 0.929 \\
-0.3 & 3.136 & 1.854 & 1.527 & 1.334 & 1.241 & 1.169 & 1.107 & 1.055 \\
-0.2 & 3.425 & 2.090 & 1.728 & 1.559 & 1.462 & 1.394 & 1.332 & 1.291 \\
-0.1 & 3.849 & 2.401 & 2.040 & 1.886 & 1.790 & 1.719 & 1.680 & 1.644 \\
0 & 4.421 & 2.848 & 2.470 & 2.310 & 2.237 & 2.204 & 2.168 & 2.135 \\
0.1 & 5.171 & 3.411 & 3.063 & 2.955 & 2.888 & 2.877 & 2.857 & 2.850 \\
0.2 & 6.289 & 4.325 & 3.961 & 3.913 & 3.888 & 3.874 & 3.940 & 3.938 \\
0.3 & 8.086 & 5.724 & 5.397 & 5.413 & 5.401 & 5.472 & 5.612 & 5.719 \\
0.4 & 12.358 & 8.748 & 8.442 & 8.689 & 8.893 & 9.173 & 9.427 & 9.645 \\
0.49 & 41.485 & 30.391 & 30.180 & 31.282 & 32.339 & 33.676 & 34.777 & 35.990 \\
\hline \hline$\backslash m$ & 9 & 10 & 11 & 12 & 13 & 14 & 15 & 16 \\
\hline-0.49 & 0.854 & 0.821 & 0.787 & 0.762 & 0.735 & 0.711 & 0.692 & 0.672 \\
-0.4 & 0.885 & 0.853 & 0.824 & 0.797 & 0.774 & 0.752 & 0.733 & 0.714 \\
-0.3 & 1.022 & 0.984 & 0.955 & 0.928 & 0.910 & 0.888 & 0.876 & 0.855 \\
-0.2 & 1.266 & 1.228 & 1.199 & 1.172 & 1.153 & 1.141 & 1.117 & 1.109 \\
-0.1 & 1.625 & 1.597 & 1.571 & 1.547 & 1.530 & 1.505 & 1.501 & 1.482 \\
0 & 2.114 & 2.105 & 2.097 & 2.087 & 2.066 & 2.070 & 2.067 & 2.061 \\
0.1 & 2.843 & 2.855 & 2.858 & 2.857 & 2.872 & 2.886 & 2.909 & 2.904 \\
0.2 & 3.973 & 4.007 & 4.030 & 4.059 & 4.104 & 4.148 & 4.197 & 4.229 \\
0.3 & 5.792 & 5.885 & 6.008 & 6.125 & 6.181 & 6.289 & 6.364 & 6.468 \\
0.4 & 9.847 & 10.114 & 10.369 & 10.532 & 10.737 & 10.968 & 11.170 & 11.339 \\
0.49 & 37.112 & 38.193 & 39.272 & 40.218 & 41.149 & 42.089 & 42.989 & 43.799 \\
\hline & & & & & & & & \\
\hline
\end{tabular}


Table 2. Rejection frequencies for different asymptotics

\begin{tabular}{|c|c|cccccccc|}
\hline$T$ & $m$ & 1 & 2 & 3 & 4 & 5 & 6 & 7 & 8 \\
\hline 64 & $t_{2 m}$ & 0.052 & 0.053 & 0.056 & 0.063 & 0.069 & 0.076 & 0.084 & 0.093 \\
64 & $N(0,1)$ & 0.188 & 0.126 & 0.112 & 0.104 & 0.104 & 0.107 & 0.111 & 0.116 \\
\hline 256 & $t_{2 m}$ & 0.049 & 0.047 & 0.049 & 0.047 & 0.048 & 0.051 & 0.050 & 0.051 \\
256 & $N(0,1)$ & 0.186 & 0.118 & 0.095 & 0.084 & 0.076 & 0.073 & 0.069 & 0.068 \\
\hline & $m$ & 9 & 10 & 11 & 12 & 13 & 14 & 15 & 16 \\
\hline 64 & $t_{2 m}$ & 0.099 & 0.107 & 0.116 & 0.124 & 0.132 & 0.141 & 0.150 & 0.157 \\
64 & $N(0,1)$ & 0.120 & 0.129 & 0.136 & 0.144 & 0.151 & 0.158 & 0.164 & 0.172 \\
\hline 256 & $t_{2 m}$ & 0.050 & 0.052 & 0.052 & 0.054 & 0.054 & 0.056 & 0.058 & 0.058 \\
256 & $N(0,1)$ & 0.067 & 0.064 & 0.066 & 0.067 & 0.066 & 0.067 & 0.068 & 0.067 \\
\hline
\end{tabular}

Table 3. Rejection frequencies, $\delta=0, \phi=0$

\begin{tabular}{|c|c|c|c|c|c|c|c|c|c|c|c|}
\hline$T$ & $m$ & 1 & 2 & 3 & 4 & 5 & 6 & 7 & 8 & & \\
\hline 64 & & 0.068 & 0.084 & 0.094 & 0.102 & 0.109 & 0.112 & 0.114 & 0.116 & & \\
\hline 256 & & 0.055 & 0.060 & 0.067 & 0.072 & 0.074 & 0.075 & 0.075 & 0.077 & & \\
\hline 1024 & & 0.050 & 0.056 & 0.059 & 0.062 & 0.062 & 0.063 & 0.063 & 0.063 & & \\
\hline & $m$ & 9 & 10 & 11 & 12 & 13 & 14 & 15 & 16 & $T$ & $\theta$ \\
\hline 64 & & 0.118 & 0.119 & 0.118 & 0.119 & 0.120 & 0.120 & 0.121 & 0.125 & 64 & 0.124 \\
\hline 256 & & 0.078 & 0.080 & 0.080 & 0.080 & 0.081 & 0.081 & 0.082 & 0.082 & 256 & 0.096 \\
\hline 1024 & & 0.063 & 0.063 & 0.064 & 0.063 & 0.065 & 0.064 & 0.063 & 0.065 & 1024 & 0.076 \\
\hline
\end{tabular}


Table 4. Rejection frequencies, $\delta=-0.3, \phi=0.5$

\begin{tabular}{|c|c|c|c|c|c|c|c|c|c|c|c|}
\hline$T$ & $m$ & 1 & 2 & 3 & 4 & 5 & 6 & 7 & 8 & & \\
\hline 64 & & 0.036 & 0.034 & 0.031 & 0.030 & 0.028 & 0.027 & 0.027 & 0.027 & & \\
\hline 256 & & 0.042 & 0.032 & 0.030 & 0.028 & 0.025 & 0.025 & 0.023 & 0.024 & & \\
\hline \multirow[t]{2}{*}{1024} & & 0.043 & 0.043 & 0.039 & 0.037 & 0.034 & 0.033 & 0.035 & 0.033 & & \\
\hline & $m$ & 9 & 10 & 11 & 12 & 13 & 14 & 15 & 16 & $T$ & $\theta$ \\
\hline 64 & & 0.027 & 0.027 & 0.028 & 0.029 & 0.030 & 0.032 & 0.033 & 0.036 & 64 & 0.031 \\
\hline 256 & & 0.021 & 0.020 & 0.020 & 0.018 & 0.018 & 0.018 & 0.018 & 0.018 & 256 & 0.022 \\
\hline 1024 & & 0.033 & 0.032 & 0.030 & 0.030 & 0.029 & 0.028 & 0.028 & 0.029 & 1024 & 0.026 \\
\hline
\end{tabular}

Table 5. Rejection frequencies, $\delta=0.3, \phi=-0.5$

\begin{tabular}{|c|c|c|c|c|c|c|c|c|c|c|c|}
\hline$T$ & $m$ & 1 & 2 & 3 & 4 & 5 & 6 & 7 & 8 & & \\
\hline 64 & & 0.111 & 0.160 & 0.186 & 0.202 & 0.211 & 0.217 & 0.222 & 0.226 & & \\
\hline 256 & & 0.068 & 0.090 & 0.099 & 0.107 & 0.112 & 0.113 & 0.115 & 0.117 & & \\
\hline 1024 & & 0.054 & 0.060 & 0.061 & 0.062 & 0.064 & 0.063 & 0.063 & 0.063 & & \\
\hline & $m$ & 9 & 10 & 11 & 12 & 13 & 14 & 15 & 16 & $T$ & $\theta$ \\
\hline 64 & & 0.229 & 0.231 & 0.232 & 0.232 & 0.233 & 0.231 & 0.231 & 0.233 & 64 & 0.244 \\
\hline 256 & & 0.118 & 0.119 & 0.120 & 0.122 & 0.123 & 0.124 & 0.124 & 0.125 & 256 & 0.145 \\
\hline 1024 & & 0.065 & 0.065 & 0.064 & 0.064 & 0.066 & 0.065 & 0.065 & 0.065 & 1024 & 0.101 \\
\hline
\end{tabular}

